\title{
Criminalidad y desajustes de las políticas de seguridad: un panorama de América Latina del post-Guerra Fría
}

\author{
Criminality and misguided security policies: an overview \\ of Latin America after the Cold War
}

Jaseff Raziel Yauri Miranda • miranda.raziel@hotmail.com

UNIVERSIDAD DEL PAÍS VASCO/EUSKAL HERRIKO UNIBERTSITATEA

UPV/EHU

Recibido: 28/03/2016

Aceptado: 07/11/2016

\section{Resumen}

En América Latina, la criminalidad se ha convertido en un fenómeno de primer orden desde el fin de la Guerra Fría. La creciente atención, sin embargo, no ha sido acompañada por una reducción de su incidencia y por un eficaz tratamiento. Las inconsistencias y fracasos para tratar el crimen también habrían tenido que ver con la propia calidad de las políticas públicas de seguridad. En ese sentido, el artículo cuestiona si tales políticas se han presentado "desajustadas» tanto en sus bases como en sus modos de acción frente a la criminalidad. Por lo tanto, en primer lugar se analizan cuáles han sido las bases teóricas y cognitivas que han sostenido las políticas públicas de cara a la criminalidad. En segundo lugar, se analizan las respuestas y transformaciones que las propias políticas de seguridad han tenido en América Latina. De manera general, las conclusiones extraen el carácter histórico y sociopolítico de las limitaciones y características que la acción pública ha tenido frente al delito, lo que confirma que los desajustes en las políticas también han contribuido a la expansión de la criminalidad en las últimas décadas.

Palabras clave: criminalidad, políticas públicas, seguridad pública, América Latina.

\section{Abstract}

In Latin America, crime has become a relevant issue since the end of the Cold War. The increased attention on the phenomena, however, has not been followed by a reduction of its incidence and by an effective treatment. The inconsistencies and failures to deal with crime would have been related to the very quality of public security policies. In that sense, the article questions whether these policies have been misguided both in their bases and in their modes of action against criminality. Therefore, we first analyze the theoretical and cognitive principles of security policies in the face of crime. Secondly, we analyze the responses and transformations of these policies in Latin America. The conclusions draw the historical, political and social nature of the limitations and features that the security policies have had against crime, confirming that policy mismatches have also contributed to the expansion of criminality during the last decades.

Key words: criminality, public policies, public safety and security, Latin America. 


\section{INTRODUCCIÓN}

La creciente complejidad de las formas del crimen en América Latina, identificada sobre todo a partir del fin de la Guerra Fría, es uno de los fenómenos que más ha llamado el interés de politólogos, sociólogos, periodistas y estudiosos de la región en las últimas décadas. Su magnitud, que se refleja en los datos de victimización y violencia en distintos países ha sido objeto extensivo de análisis académicos. Al mismo tiempo, la criminalidad ha servido como generadora de miedos y de un estigma sensacionalista en diversos programas comunicativos. Sin embargo, la gran atención que tiene como objeto el crimen parece ser discrepante ante los planteamientos y efectos de las políticas públicas en seguridad que se enfocaron en este fenómeno. Es decir, se habría producido un desajuste entre el fenómeno de la criminalidad y el tratamiento dado por estas políticas públicas, en términos de bases y resultados, pese a que el fenómeno tenga relevancia en la historia reciente de la región.

En la Historia se menciona que los eventos inacabados o que son traumáticos para una colectividad tienden a no ser encuadrados fácilmente dentro de la memoria histórica. Siendo así no es raro que, en la literatura producida sobre la historia política del post-Guerra Fría, el tema de la criminalidad sea aún un tema incipiente. Probablemente porque la criminalidad reciente es considerada como un fenómeno en continuo desarrollo, o porque la historiografía está marcada por el peso que todavía ejercen los períodos de violencia política de los regímenes dictatoriales del pasado. ${ }^{1}$ Sin embargo, es un hecho que el crimen ya cumple un papel notable en la historia reciente de los países de la región. La violencia del crimen de las últimas décadas es tan grave como la violación de los derechos y el terrorismo de Estado cometidos en algunos países durante las décadas de 1960, 1970 o 1980. ${ }^{2}$ Y sus distintas formas de violencia gozan de una situación que se agrava incluso por la forma con que los mismos países tratan y reaccionan frente a la criminalidad.

En los años 1990, los homicidios en la región, por ejemplo, representaron casi el 30\% de las cifras globales, siendo que los latinoamericanos constituían el 9\% de la población mundial (UNODC, 2011). Al mismo tiempo, se ha mantenido un promedio de más de 20 homicidios por cada 100 mil habitantes desde esa misma década hasta los últimos años. Para tener una idea, esa misma tasa se situaba en 6,2 a nivel global en el año 2012 (UNODC, 2013). Tal fatalidad no dejó de ser un tipo de violencia política, ya que en un panorama donde se produce demanda de más seguridad, el énfasis en modelos mal articulados y basados en la represión ha perjudicado la seguridad y la consolidación democrática en muchos países. En este sentido es pretencioso reducir las características y la comprensión que se tiene del término «democracia», pero para consolidar este sistema fue necesario (y se necesita) ir más allá de elecciones periódicas basadas en el voto popular, con el fin de institucionalizar

\footnotetext{
1 Para una revisión de la historiografía sobre algunas dictaduras en el continente, véase Ramírez, 2012; y, Quiroga, 2005.

${ }^{2}$ Un ejemplo sobre la conceptualización de violencia de Estado y la relación entre «violencia represiva» y «violencia preventiva» a partir de la experiencia dictatorial uruguaya puede ser apreciado en Padrós, 2012:2.
} 
funciones públicas junto a la ciudadanía, de modo a no perder de vista la pluralidad y las demandas sociales de la región incluso en materia de seguridad colectiva.

Desde finales del siglo XX, si las transiciones a la democracia o la búsqueda del fortalecimiento de ese sistema, junto con la expansión del fenómeno de la criminalidad, han sido marcos comunes a los países de América Latina, no obstante, se pueden observar peculiaridades en las respuestas de las administraciones nacionales. Por un lado se identifican experiencias que produjeron resultados considerables sobre el crimen, sea en términos de contener su incidencia o de conjugar nuevos actores sociales en las últimas décadas. Pero en su conjunto, en América Latina han persistido inconsistencias a pesar de los esfuerzos gubernamentales para frenar la criminalidad (UNODC, 2013), tal como será visto adelante.

Por tales problemas, habría que dirigir la mirada justamente a las políticas de seguridad pública ya que la criminalidad aún es un reto para las administraciones de la región. Dentro de las políticas públicas, habría características que también han contribuido a la baja calidad de respuesta ante la realidad criminal. Es decir, debido a las «inconsistencias» 0 «fracasos» en las políticas públicas de las últimas décadas, el artículo cuestiona si estas se han presentado "desajustadas» tanto en términos de sus propias bases cognitivas ante el avance del crimen, como en la intervención para tratar este fenómeno. Siendo así, para verificar esos desajustes entre políticas públicas y el tratamiento dado a la criminalidad, en la primera sección del artículo se analizan cuáles han sido las bases teóricas que han fundamentado las políticas públicas frente al crimen. En la segunda sección del artículo, junto a esos modelos se analizan cuáles han sido las respuestas generales o modos de intervención de las políticas frente a la criminalidad en las últimas décadas.

El artículo capta las transformaciones y coyuntura histórica de las políticas de seguridad en las últimas décadas en América Latina. El marco temporal específico cubre el inicio de la década de 1990, época de transiciones democráticas y de inicio del panorama actual del Post-Guerra Fría, hasta mediados de la segunda década del actual siglo. Por tal motivo, el análisis cualitativo en las secciones recurre a aportes teóricos interdisciplinares desde el campo de la Historia y de las Ciencias Sociales. Al mismo tiempo, se consideran estudios de entidades internacionales, como la Oficina de las Naciones Unidas contra la Droga y el Delito, UNODC, el Latinobarómetro, el Programa de las Naciones Unidas para el Desarrollo, PNUD, bien como fuentes secundarias realizados por estudiosos de la seguridad en distintos países de la región. El presente artículo no pretende realizar una compilación de las experiencias de las políticas en cada país o formular las mejores hipótesis para la gestión de la criminalidad. No obstante, la exposición y análisis general de las políticas de seguridad pública en un sentido holístico, junto a sus principios cognitivos y a su manejo, es un esfuerzo esencial para todo estudioso o interesado en la realidad latinoamericana. 


\section{BASES TEÓRICAS E INTERPRETATIVAS DE LAS POLÍTICAS PÚBLICAS}

En las últimas décadas, aunque América Latina haya sido una región pacífica en términos de conflictos bélicos interestatales, la criminalidad de ámbito interno y transnacional ha amplificado la violencia. Los estudios en la región se han referido al crimen como un fenómeno derivado de violencia interpersonal y sociopolítica, siendo tipificada en cada país según criterios propios asociados a delitos como robo, secuestro, homicidio, latrocinio, violencia de género, entre otros. En ese conjunto, un trazo común en la región es que muchos de esos delitos se relacionan con cuestiones como la delincuencia callejera y el tráfico de drogas (PNUD, 2014). Sin embargo, la heterogeneidad de ámbitos políticos y legales de todos los países hace que una única definición de criminalidad no sea exacta y suficiente.

La construcción de datos «duros» sobre la criminalidad, a cargo de las policías, los sistemas de Justicia y Salud entre los países, también es fragmentada para definir y encuadrar su incidencia. Por otra parte, los datos «blandos», índices de percepción y encuestas de victimización, han complementado el entendimiento y la realidad del crimen, aunque también sufren desvíos. La comparación entre las cifras de los países ha sido una tarea ardua, sobre todo al inicio de los años 90 , donde los métodos de colecta y procesamiento de información eran más heterogéneos. Desde finales del siglo pasado, asociaciones multilaterales, como la UNODC, el PNUD y el Latinobarómetro han intentado reunir alguno de esos dos tipos de datos desde una perspectiva internacional. Estos estudios se han beneficiado de la mejora de compilación de datos nacionales y han posibilitado ofrecer panoramas más integrados sobre el crimen en la región, sobre todo de homicidios, delito cuya percepción e índice es más fiable, y cuya tasa sigue siendo elevada en la región. Véase la Figura 1.

Figura 1. Homicide rates, by sub-region (1995-2012)

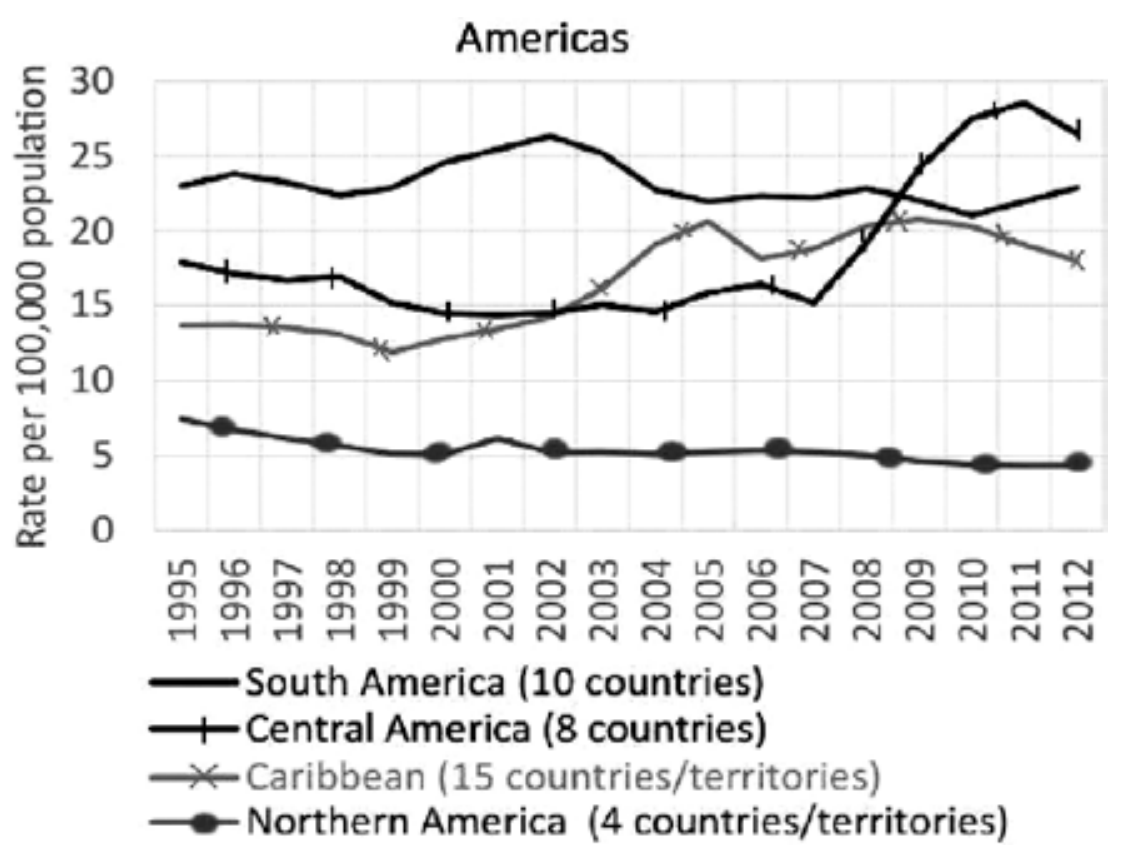

Fuente: UNODC, 2013. 
La figura muestra la evolución de la tasa de homicidios intencionales (excluyendo aquellos cometidos en guerras, suicidios, legítima defensa e intervenciones legales), para cada 100 mil habitantes en sub-regiones de América. Según el mismo informe, esta tasa se situaba en 6,2 en 2012 a nivel global, último año de la medición, dando una idea del panorama de un tipo de crimen que sigue acometiendo millares de vidas a cada año, sobre todo de perfil masculino y joven (entre 15 a 35 años). ${ }^{3}$ A esto se suma que la criminalidad (incluyendo prácticas como los homicidios) es un fenómeno multifacético que sigue siendo difícil de entender en sus motivos y causalidades. Se menciona la «pobreza (económica) como génesis de la delincuencia», aunque esta causalidad no sea directa o no se sustente en determinados «ámbitos geográficos o marcos temporales» (Espinoza, 2008:21). El aumento de riqueza latinoamericana y la menor desigualdad económica, como la ocurrida entre 2000 y 2010, aunque no deban ser desestimuladas, tampoco fueron suficientes para apaciguar las tasas de criminalidad en relación a periodos anteriores (CEPAL, 2015). Por otra parte, el entendimiento sobre el crimen se agravó después del conflicto bipolar a finales del siglo xx, cuando la criminalidad ganó «plasticidad» y difusión, llegando a penetrar en diversos países y estratos sociales. Esto marcó el inicio de un panorama donde los preceptos y funciones de los Estados se diluyeron a medida que otros actores ganaron terreno en la globalización mundial, «compitiendo incluso en las esferas del crimen» (Garland, 1996: 445).

En la acelerada globalización económica del post-Guerra Fría, con acceso a más recursos y al crecimiento de flujos de capital, actividades consideradas ilícitas por los ordenamientos estatales también han tenido numerosas chances de entrelazarse con nuevos mercados. El crimen y la violencia adyacente a esas actividades pasaron a desafiar un tipo de Estado que funciona por principios tardíos o reactivos. Basta recordar que los convenios internacionales promovidos para reaccionar frente a delitos como lavado de dinero, narcotráfico y corrupción internacional, ejemplifican la creciente magnitud del fenómeno de la criminalidad que ignora los territorios nacionales y se mueve entre sus legislaciones. ${ }^{4}$

Ante la creciente complejidad del fenómeno del crimen, incluso desde la década de 1970 ya se pensaba en la necesidad de desarrollar nuevos modelos de seguridad. Por ejemplo, uno en el cual la policía fuese más allá del uso del arma y del enfoque sobre el hecho delictivo. El sociólogo Daniel Cerqueira (2007) cita a Blumstein, Cohen y Nagin (1978), y a Spelman y Brown (1984), como estudiosos del mundo anglosajón que cuestionaron el modelo tradicional de lucha contra el crimen, marcado por estrategias enfocadas en arrestar y neutralizar delincuentes (Cerqueira y Carvalho, 2007:13). A finales del siglo pasado, en América Latina también se hablaba de remodelar una policía que se orientaba a la simple supresión de conflictos. Era esencial cogitar prácticas intersectoriales, sobre todo con ac-

\footnotetext{
3 Para datos completos de la evolución de las cifras de homicidios desde el año 2000 en todos los países de América Latina, véase índice técnico en UNODC, 2013. Para datos sobre perfil de la población víctima de homicidios, bien como datos sobre otros delitos, véase los informes del PNUD, 2014 y PNUD, 2016.

${ }^{4}$ A saber, la Convención Interamericana contra la Corrupción de 1997; la Convención de Viena de 1988, que tipifica como delito el lavado de dinero internacional; la Conferencia de Palermo en el año 2000, que aprobó la Convención de las Naciones Unidas Contra la Delincuencia Organizada; y la Convención de Mérida de 2003, por la cual quedó establecida la Convención de las Naciones Unidas contra la Corrupción.
} 
ciones junto a las comunidades en las periferias de grandes ciudades. También era necesario pensar en sectores como la cultura o la educación, los cuales estaban fuera del rango de actividades del profesional uniformado. En teoría, todo esto demandaba una remodelación interna del trabajo de la propia policía, y exigía una política de seguridad hacia otras direcciones o diseños (Cerqueira y Carvalho, 2007: 14-5).

A pesar de esas demandas, en América Latina, los programas de acción política en materia de criminalidad se han enfrentado a una inercia en el modo de operatividad y muchas veces han producido resultados mínimos para frenar el crimen. Y tales limitaciones, tanto en lo que dice respecto a la remodelación de las policías como en la conjugación con factores extra-policiales también se han vinculado al uso y comprensión que se hizo sobre las políticas públicas (Lagos y Dammert, 2012:55). Por lo tanto, es esencial esclarecer qué se entendía o cuáles han sido las bases teóricas de las «políticas» a medida que avanzaba el crimen.

Las políticas públicas se comprendían, básicamente, como un conjunto de acciones racionales del Estado, el cual se enfocaba en la resolución o modificación de determinado problema, por ejemplo, en la reducción de los índices de criminalidad. Luis Antonio F. De Souza (2011), al analizar la literatura de las ciencias sociales latinoamericanas, identificó tres modelos de comprensión de influencia anglosajona, cuyos preceptos tienen como objeto el Estado y los procesos de decisión en las políticas. Dos de estos modelos, de vertientes top-down y bottom-up, pueden ser así resumidos:

Tenemos que entrar en el llamado análisis bottom-up. Su importancia ha crecido desde la década de 1980 con la investigación de Michael Lipsky (1980), que llamó la atención para el hecho de que los modelos de análisis de las políticas públicas eran top-down, es decir, excesivamente concentrados en actores de toma de decisiones, que elaboran determinada política. Sin dejar de lado el uso de modelos de análisis top-down, las investigaciones pasaron a utilizar también análisis bottom-up, las cuales comparten tres características: a) estudiar determinada política pública a partir de las actividades de sus implementadores, en oposición a la excesiva concentración de los estudios sobre los gobiernos, tomadores de decisiones y actores que se sitúan en las esferas «centrales»; b) centrar el análisis sobre la naturaleza del problema al cual la política pública busca responder; c) describir y analizar la implementación de redes. (..) Modelos de análisis bottom-up pueden estar sujetos a críticas cuanto a su capacidad explicativa, pero necesitan ser más examinados en nuestra realidad, [...] por no ignorar y asumir la complejidad de la política pública como algo que necesita ser explicado sobre todo en su implementación [...]. (Celina Souza, in Souza, A. F., 2011:5).

De acuerdo con Souza, un tercer modelo de análisis, y que no excluye necesariamente los anteriores, es el «ciclo vivo» de las políticas públicas. En este último se opta por un análisis secuencial donde se produce una articulación entre distintas fases: «definición de la agenda, identificación de alternativas, evaluación y selección de opciones, formulación, 
implementación y evaluación» (Souza, 2011:6) en un movimiento circular continuo y de constante redefinición de sus etapas. Se trata, por lo tanto, de una matriz teórica que cubre las arenas políticas o grupos de actores que intentan convencer el sistema político sobre la importancia o necesidad de determinada acción pública (Souza, 2011: 6). En este modelo, las demandas de las arenas se convierten en políticas a través de las conocidas "ventanas de oportunidad». Es decir, momentos de mayor permeabilidad de la agenda, en los que determinados «problemas y soluciones» transbordan de un ámbito particular, o de ciertos grupos de interés, hacia el ámbito público (Kingdon, 1984). En esta matriz, se consideran los «actores» que cumplen roles específicos, tales como los formuladores de decisiones, los implementadores y los evaluadores, en un ciclo completo y que se retro-alimenta y donde determinada institución, como la policía, puede cumplir más de un rol. Por tales características, el modelo del «ciclo vivo», más que una invención dentro las ciencias sociales es un intento de abarcar aspectos de las políticas públicas desde ángulos racionalistas, institucionales/normativos y hasta simbólicos. Todo ello en un sentido de corregir el «ciclo» ante determinados errores o déficits inherentes a cada ángulo.

A su vez, los modelos de carácter bottom-up y del «ciclo vivo" se interrelacionaron con los principios de la «Nueva Administración Pública» (NAP) o "gerencialismo», la cual pasó a corroborar principios de eficiencia y flexibilidad administrativa a través de formación de metas, desarrollo e implementación de planes, y evaluación por resultados (Meny y Thoenig, 1992; Muller, 2002; Subirats et al, 2008; Valenti y Flores, 2009). Por tal interrelación, hubo una incorporación de herramientas administrativas que ya habían ganado terreno en las arenas privadas y corporativas desde los años 80, las cuales luego transbordaron hacia el ámbito estatal al aprovechar una ventana de oportunidad: la crisis fiscal y las privatizaciones de sectores públicos en América Latina en los años 1990. El desgaste de la máquina pública en aquel momento se convirtió en un problema de primer orden en las agendas neoliberales del post-Guerra Fría. De tal modo, las administraciones estatales necesitaban reformas urgentes conforme recomendaba, por ejemplo, el «Consejo Director del Centro Latinoamericano de Administración para el Desarrollo» (CLAD) en 1988. ${ }^{5}$

Los principios de eficiencia y flexibilidad que perfilaron las primeras reformas gerenciales de las administraciones públicas, como la ocurrida en 1993 en Chile y en 1995 en Brasil, ganaron preponderancia al comienzo de los años 2000. En estos años se profundizó el discurso de llevar a cabo una gestión preocupada por el establecimiento de metas, de promover alianzas con el sector privado, por distribuir competencias y por mencionar implementadores, evaluadores y, sobre todo, usuarios/ciudadanos en las políticas públicas. Este discurso, por lo tanto, se aleja de una centralización y de un movimiento más unidireccional encontrado en el modelo top-down. Sin embargo, la implementación de acciones integrales, que recuerdan a los modelos bottom-up y del «ciclo vivo», fueron difíciles de re-

\footnotetext{
${ }^{5}$ Chile constituiría una excepción a tales recomendaciones, ya que se anticipó a la naturaleza de las recomendaciones del CLAD al haber implementado algunos parámetros gerenciales en la administración pública aún en la época del régimen militar de Pinochet (Orrego, 1998).
} 
producir o de incorporarse a la acción gubernamental en el presente siglo. Los motivos serán mejor explicados en la siguiente sección, pero se adelanta que las agendas y arenas de toma de decisiones de los países se encontraban sometidas a una administración pública que, según Paes de Paula, «se equivocaba al distinguir las políticas públicas (policy) de lo político (polity) y se basaba en una polaridad típica de esferas privadas, en las cuales existe una dicotomía entre administración vs. política» (Paes de Paula, 2005:46). De modo que, entre la formulación y la implementación de las políticas, se produjeron grietas, cambios e interpolaciones que no estaban previstas en el momento de definición de las agendas.

Al mismo tiempo, en el ámbito de la criminalidad en América Latina, las ideas que sirvieron para desarrollar las políticas progresaron a un ritmo lento porque, según Lucía Dammert y John Bailey (2005), sus ajustes fueron programados a través de: a) una tradición intelectual desconectada de un énfasis "práctico» y empírico; b) una experiencia extranjera, que pretendía transferir experiencias y las presuposiciones de realidades distintas; y, c) poco debate público, observado sobre todo en los momentos en que los políticos ensayaron propuestas para sus proyectos electorales. Citando Jaymes Q. Wilson, Bailey muestra que:

El conocimiento de las ciencias sociales no se traduce automáticamente en conocimiento de políticas que sean prácticas. El conocimiento [...] puede ser útil en resolver ciertos problemas y en buscar conexiones causales. En cambio, la prescripción política requiere un mayor conocimiento sobre cómo intervenir adecuadamente en el entorno para conseguir los resultados esperados. Es decir, intenta dar respuestas con instrumentos específicos de la política (ej. Fuerzas de policía) y medidas (ej. Patrullas motorizadas en ciertos barrios) sobre crímenes específicos (ej. Robo armado). Esto ante situaciones de escasez y recursos, y ante la demanda de la sociedad de obtener resultados,[...] para dar respuestas a los problemas (Dammert y Bailey, 2005:366-67).

Por lo tanto, ya sea por ignorar factores exógenos para concebir las políticas públicas o por limitaciones inherentes a los análisis científicos, el hecho es que las políticas tardaron a incorporar preceptos epistémicos y pragmáticos más dinámicos, abiertos y retroalimentares. Esto es, las políticas fueron muchas veces tardías o reactivas, tuvieron poco debate público y se acercaron más a modelos top-down que a etapas secuenciales o del «ciclo vivo». Mientras tanto, el crimen se constituyó como un problema público en América Latina y que afecta, hasta la fecha, diversos espacios de convivencia colectiva. Las formas de violencia de connotaciones más privadas, como la violencia doméstica, de género, la corrupción de 'cuello blanco', entre otras, aunque no menos importantes, cedieron predominio, por ejemplo, al crimen denotado en las calles, al tráfico de drogas y a los homicidios (Lagos y Dammert, 2012: 45). Consecuentemente, cabe expresar, de manera general, qué respuestas han dado las políticas públicas teniendo en cuenta este panorama lleno de obstáculos para las comunidades y países de la región. 


\section{RESPUESTAS Y TRANSFORMACIONES DE LAS POLÍTICAS PÚBLICAS}

Siguiendo la línea histórica que dice respecto a las políticas sobre la criminalidad en América Latina, es necesario comprobar el pasado de las acciones que buscaron garantizar el orden y la seguridad de las personas. En esa tarea, se observa que su incidencia, incluyendo la percepción y el temor a la criminalidad, han crecido durante desde la década de 1990 (Latinobarómetro, 2011). Últimamente, la «delincuencia/seguridad pública» se presenta como el "problema más importante» dentro de los países para 22,8\% de los habitantes, por encima del desempleo (para 16,2\%), mal desempeño de la economía (8,2\%), entre otros (Latinobarómetro 2015). Sin embargo, se recuerda que la criminalidad y la violencia han sido recurrentes incluso antes de ese período pese a que no hayan sido tratados como una prioridad en la agenda de los países latinoamericanos.

En los años 70 y 80 del siglo pasado, muchos países latinoamericanos pasaron por períodos autoritarios, por conflictos sociopolíticos internos o por la supresión de garantías civiles. En ellos fueron instaurados verdaderas «prácticas y formas de control sobre la ciudadanía, las cuales exacerbaron la simple coerción política» (Martínez, 1990:18). Durante este período, la seguridad y el orden público se encontraban dentro de los programas ideológicos presentes en las «Doctrinas de Seguridad Nacional» que, según Nilson Borges (2003), centraron sus esfuerzos en la defensa contra las amenazas internas al sistema político e institucional. Así, el ciudadano común «cedió» su libre ejercicio de derechos porque, según la óptica estatal, se vivía un periodo de emergencia, en el cual los crímenes que más destacaban eran aquellos derivados de la disidencia política. Por lo tanto, según la lógica de la bipolaridad mundial, la seguridad pública estaba más condicionada a una «seguridad nacional» que a una «seguridad ciudadana».

Al llegar la década de 1990, después de la Guerra Fría y con el colapso o la transición de regímenes autoritarios a democracias renacientes, en muchos países del continente se observa que la violencia política, incluyendo el terrorismo de Estado, ha sido sustituida por el crimen de la calle y por los delitos en el espacio público. Enrique Oviedo (2002) advierte que el aumento de la percepción de la violencia en la década de 1990, en parte, se debe a la función de los medios de comunicación, los cuales han dirigido su enfoque del entorno político hacia las acciones delictivas en los espacios colectivos. Según Oviedo, la violencia no es un fenómeno nuevo, sino, paradójicamente, uno de los logros de los nuevos gobiernos y de las libertades de expresión ha sido convertir el problema de la seguridad en una cuestión pública. Algo que, a su vez, desacreditó, de cierta forma, el sistema democrático ante la opinión general de los ciudadanos. ${ }^{6}$

\footnotetext{
${ }^{6}$ Como menciona Lagos en Lagos y Dammert, 2012:58, «Es la democracia la que la visibiliza al punto que le da a cada cual posibilidades de demandar seguridad como un derecho. Esto a su vez tiene impacto en la imagen de las instituciones de la democracia y su gobernabilidad».
} 
Incluso una de las razones de la actual relativa desconfianza en la democracia, ya que para $34,5 \%$ de los latinoamericanos «en algunas circunstancias es preferible un régimen autoritario" o "da lo mismo un régimen democrático que uno no democrático» (Latinobarómetro, 2015), puede ser causada por el mal entendimiento que se tiene sobre el crimen. Es decir, a partir del evidente aumento de la criminalidad a finales del siglo xx, también se dio lugar a una interpretación de que, durante los períodos autoritarios, la sociedad era más segura debido a la acción represiva y al uso de la fuerza que por el contexto de la época. Tal contexto se reduciría a un sentido donde las policías y fuerzas armadas son "expertos» en el combate de la delincuencia, y deja de lado factores como la formación de mega-ciudades, el narcotráfico internacional, la forma de elaboración de estadísticas, la percepción y el papel de los medios de comunicación, entre otros.

Dentro de esos factores, una las características principales del fenómeno de la criminalidad en las últimas décadas fue su «urbanización» y posterior «ruralización». En la década de 1990, las principales áreas metropolitanas adquirieron indicadores críticos de violencia, lo que convirtió a América Latina en la segunda región más violenta del mundo, siendo sólo superada por las regiones donde se producían conflictos bélicos (Buvinic y Morrison, 1999:3). Ya en la década de 2000, la "geografía del crimen» mostró su movilidad hacia ciudades intermediarias. En Chile y Colombia, por ejemplo, los índices de criminalidad de ciudades como Iquique y Antofagasta, o como Cali y Medellín, superaron durante varios años al de las regiones metropolitanas de Santiago y Bogotá (Caruso, H. et al, 2007:146). En los últimos años, también se ha constatado cierto desplazamiento de homicidios y otros crímenes a áreas de expansión agrícola, como en el norte de Brasil, o a regiones fronterizas y de la costa central del Pacífico en México (UNODC, 2013; Mexico Peace Index Report, 2015). Mientras tanto, la violencia en América Latina diversificó y perfeccionó sus mecanismos de acción, tales como el narcotráfico internacional, el secuestro relámpago y las pandillas de las calles, incluyendo la aparición de nuevos actores, como los sicarios y las hermandades del crimen en Centroamérica, penetrando, por lo tanto, en todos los dominios de la vida urbana y en inúmeras regiones incluso lejos de las grandes ciudades.

Al mismo tiempo, se han formado lugares de presencia limitada por parte del Estado (Maihold y Córdova, 2014) y las sociedades latinoamericanas se han sentido más inseguras, exigiendo, así, respuestas rápidas y eficaces de los gobiernos. A su vez, los gobiernos de turno prometieron soluciones circunstanciales, "milagros» que requerían respuestas de largo alcance mientras se presionaban la policía y la Justicia para producir resultados a corto plazo. No obstante, en el panorama general hasta las primeras décadas del siglo xxi, los gobiernos no pretendieron asumir el coste político e institucional que implicaría la aplicación de nuevas reformas, como en el ámbito de las policías, y así hacer frente a la inseguridad (Dammert, 2014). Hubo una ausencia de esfuerzos enérgicos por parte de los gobiernos y el reto para flexibilizar la gestión de control y lucha contra la criminalidad, sobre todo la que incidía en los espacios públicos y en las ciudades. Esto también se entre- 
lazó con otros factores estructurales, como los problemas derivados de la crisis fiscal que asoló casi toda la región durante la década de 1990 y la crisis económica enfrentada por la baja de las exportaciones primarias a partir de la crisis del año 2008. Desde finales del anterior siglo, la agenda de los gobiernos centrales pasó de un énfasis a la protección de mercados y de incentivos a la productividad nacional, para un marco pautado por privatizaciones, búsqueda de dividendos financieros y atracción de capital extranjero (Dammert y Bailey, 2005: 15- 6). Y aunque esto no haya sido implementado a fondo en todos los países, sobre todo en aquellos situados en el espectro «bolivariano», tal agenda ha regresado con ímpeto en los últimos años, significando un estancamiento de las inversiones en sectores sociales que son complementarios a la seguridad pública.

Desde la década de 1990, por lo tanto, hubo un doble desafío. Uno de dimensión política, que decía respecto a la redemocratización o hacia la profundización de ese sistema, ${ }^{7}$ y otro de dimensión económica, los cuales se combinaron para agravar la comprensión y las respuestas a la criminalidad. Si por un lado, muchas de las respuestas policiales aún poseían marcas de violaciones contra las garantías civiles, por otro lado, los recursos económicos no fueron suficientes en determinados ciclos. Por consiguiente, se ha verificado que las acciones estatales han sido limitadas tanto en escala como en resultados. Ante esta situación, Guillermo O’Donnell ya había llegado a cuestionar, en la dimensión de la transición política, si estas jóvenes democracias evolucionarían de forma natural hacia democracias más consolidadas -con fuertes sistemas representativos, mayores garantías constitucionales y responsabilidad pública-, o sufrirían una muerte lenta debido a la deficiente toma de decisiones, a la corrupción y a la violencia (O’Donnell, 1994).

Heredando poca capacidad para diseñar e implementar decisiones programáticas, las instituciones relacionadas al tratamiento de la criminalidad -a ejemplo de la policía, los tribunales de justicia, el sistema penitenciario- han quedado bajo la tutela de los métodos y grupos vinculados a la anterior represión política. La distinción entre «seguridad nacional» y «seguridad pública/ciudadana» no ha sido consolidada (Dammert, 2007:147). A su vez, en escenarios posteriores a guerras civiles, como en Guatemala y El Salvador, donde se anhelaba llegar a un estado de paz ciudadana, tal deseo ha sucumbido ante limitaciones de capacidades y recursos en las políticas gubernamentales. En el año 2008, por ejemplo, se instauró una Comisión Internacional contra la Impunidad, creada por las Naciones Unidas, en vista del fracaso de la justicia penal de Guatemala.

En América Latina como un conjunto ha habido pocas redefiniciones respecto a las políticas y a los roles de las corporaciones de seguridad.

\footnotetext{
${ }^{7}$ Con la excepción de Colombia, México y algunos países del Caribe que no han pasado por regímenes dictatoriales prolongados que terminarían en transiciones políticas. Pese a ello, esos países tuvieron que redefinir y profundizar sus «viejas» democracias (Garretón, 1997:14). Como casos opuestos, destacan: la transición abrupta argentina post-guerra de las Malvinas en 1983; las transiciones después de décadas de autoritarismo en Brasil (1964-1985), en Chile (1973-1990) y en Bolivia (1964-1982); o las guerras civiles que cesaron fuego en Honduras (1991), en El Salvador (1992), y en Guatemala (1996).
} 
La tendencia de las policías en la región consistió en reproducir las estrategias tradicionales para combatir el crimen, que atienden a un modelo de tipo militar, reactivo y represivo, heredero de las dictaduras militares que gobernaron en América Latina durante el siglo pasado. Sin embargo, este tipo de modelo se convierte en sí mismo como un obstáculo para la transformación, ya que carece de la flexibilidad necesaria para modernizarse y adaptarse a los desafíos traídos por la criminalidad actual (Dammert, 2007: 11).

En los países de transición política, paradójicamente, el retorno de la democracia ha generado una mayor dependencia gubernamental en relación a las policías, muchas de las cuales cometieron desvíos en el pasado autoritario. Sin embargo, este protagonismo no fue necesariamente complementado por un cambio organizacional que daría lugar a mayores niveles de profesionalidad y eficiencia de esas corporaciones. Por lo contrario, el uso excesivo de la fuerza, la corrupción y la participación en actos criminales fueron notados en muchos países de la región. Esto ha aumentado los casos de ciudadanos asesinados por la policía y viceversa, en países como Brasil y Argentina, y violación de derechos civiles, como en protestas en Ecuador y Perú. Tales violaciones son evidentes sobre todo en los procesos de detención, bien como en el tratamiento de la población reclusa (Dammert, 2007:143-162). Hubo, por lo tanto, un doble desvío en las democracias latinoamericanas: uno por hacer negligencia a los derechos del ciudadano mientras este era objetivo de la acción policial, y otro por no garantizar a las colectividades una seguridad pública y un entorno propicio para el convivio social (PNUD, 2010).

La visión de desarrollo integral del ciudadano, como sujeto que debe gozar de garantías incluso en su integridad física, no siempre ha sido explícita en la agenda de las políticas públicas de seguridad del continente. En América Latina la inseguridad se abordó sobre todo desde la perspectiva de la «ley y orden», que coloca en el centro de la gestión pública el «paradigma de contención y de represión» (Espinoza, 2008: 19). No obstante, ante los clamores de gran parte de los medios de comunicación, y ensayando acciones limitadas en las políticas, la acción policial anclada en la represión y en mayores castigos para los criminales fueron bien recibidos. En Argentina, Máximo Sozzo (2005) clasificó este aspecto como el surgimiento del "populismo punitivo». En Uruguay, las respuestas a la inseguridad colectiva también fueron guiadas por políticas esencialmente punitivas o de tolerancia cero para tratar la delincuencia (Sanseviero, 2007). Estas medidas han dejado incluso resultados negativos que el gobierno Mujica, de 2010 a 2015, no ha conseguido detener. En este lapso, pese la mejora de las condiciones generales de vida de los uruguayos, de más inversión de recursos para incorporar tecnologías en seguridad y del aumento salarial de la policía, las estadísticas de robos y homicidios no han dejado de crecer, mientras solo la mitad de estos consiguen ser clarificados. ${ }^{8}$

\footnotetext{
${ }^{8}$ La seguridad en el gobierno de Mujica, según los números. El Observador, 11 de Febrero de 2015. Disponible en: http://www.elobservador.com.uy/la-seguridad-el-gobierno-mujica-segun-los-numeros-n297965, consulta en $15 / 10 / 2016$.
} 
Otros resultados negativos se verifican en las instituciones de corrección del delito, donde se han formado verdaderas «escuelas del crimen» en los sistemas de prisión.

Sin duda, la reforma de las policías es un elemento central del proceso, pero no puede, por sí misma, prevenir y controlar la violencia y el crimen en un país. Así, por ejemplo, el aumento de la presencia policial y la represión de los delitos tienen como consecuencia un gran número de prisioneros, que no siempre son infractores o culpados. A su vez, este proceso tiene efectos negativos, como el colapso del poder judicial y del sistema penitenciario, bien como de un «olvido» de la esencia de optar también por medidas de rehabilitación. A cambio, el sistema judicial colapsado se vuelve más vulnerable a la ineficiencia, a la injusticia, a la corrupción y al abuso. Y las cárceles, infraestructuras recarias y superpobladas, se convierten en peligrosos y violentos depósitos humanos, también conocidos como escuelas del crimen (Dammert, 2007: 151).

Obviamente, una acción policial de mejor calidad o intervención contra el crimen es esencial, pero la represión con fin en sí misma y el centralismo de las policías, en nuestra opinión, se debió a una visión muy «estrecha», característica de herramientas administrativas top-down, en lugar de posicionar a las mismas como «una parte en un todo» dentro de un «ciclo vivo» de políticas públicas. Como mencionado, no es nuestro objetivo exponer los intentos de reformas en las policías, pero se resalta que los mecanismos para buscar mejores resultados en la seguridad pública también han sido fruto de la presión institucional dentro de cada Estado. Es decir, acciones en la policy han sido llevadas a cabo ya que agentes políticos y funcionarios de la administración tenían que expresar medidas para lograr algún cambio, o al menos dar a conocer que algo se estaba produciendo. En tales acciones, las herramientas gerenciales o de la «Nueva Administración Pública», mencionadas en la sección anterior, han uno de los caminos encontrados.

La influencia del campo de la «nueva gestión pública (new management) es indudable [...] Naturalmente que las autoridades públicas necesitaban hacer algo al respecto, o a lo más mostrar que estaban haciéndolo: la presentación de la Estrategia fue una moneda de cambio ante un nuevo premio por hacer las cosas. A eso reporta la estrategia, a una doble presión: una "presión desde arriba», desde el gobierno central, con nuevas ideas políticas, con clamores de eficiencia en la gestión, teniendo a la vista experiencias internacionales que son reportadas por académicos, o sea, una lógica institucional que debía reorientarse; y, por otra parte, "presiones desde abajo», es decir, por un lado, desde niveles descentralizados de gobierno que comenzaron a forjar soluciones y, por otro, de personas y organizaciones que se manifestaron dispuestas para combatir el delito y recurrieron al sector público para concretarlo (Lagos, 2012:222).

Tal como indica la última cita, por las «presiones desde abajo» y en su encuentro con las «organizaciones que se manifestaron dispuestas al cambio», se deseó una descentralización y remodelación de la administración pública para que ésta no se concentrara en los tomadores de decisiones o en los mandos de las corporaciones de seguridad. Por lo tanto, 
debido a su naturaleza y a las dificultades de tratar el fenómeno, la criminalidad necesitó considerarse, en las últimas décadas, como la derivación de un fenómeno complejo, estructurado en parámetros cuya referencia demandaba un enfoque multisectorial, y que debía incluir aspectos que recordasen más a los modelos bottom-up y del «ciclo vivo» de las políticas públicas .

Motivadas por esas necesidades de cambio e insertadas en las líneas de la «Nueva Administración Pública» desde finales de los años 1990, las políticas de seguridad pública (denominadas en algunos países por seguridad ciudadana) pasaron a ser concebidas como corrientes de acción y de información relacionadas con objetivos o metas en el área pública. Se persiguió una acción secuencial en búsqueda de resultados, que aunque tuviese la tutela del estado, también despertó el interés por aglutinar a las comunidades y al sector privado (Lahera, 2002). Por lo tanto, mientras sucedía la politización del tema de la criminalidad y se pensaban acciones programáticas, poco a poco se entendía que era necesario redefinir los roles institucionales y las herramientas que orbitaban en torno al tema de la seguridad pública.

Siendo así, al considerar también las nuevas características de la criminalidad contemporánea, con sus espacios de incidencia, la amenaza a las potestades y a la soberanía del Estado, se elaboraron "planes» y propuestas de acciones interinstitucionales para orientar la lucha contra el crimen en los países. Pese a sus diferencias, tales «Planes de Seguridad» (como el «Plan Colombia», "Argentina por la Paz», o los «Planos de Segurança Pública» brasileños y los «Planes Nacionales» de seguridad ciudadana en Chile y Perú, que se actualizan a cada cuatro o cinco años) alertaron para la modernización de las instituciones de lucha contra el crimen, estableciendo criterios gerenciales y, en algunos casos, mejorando la eficacia y resultados en esa lucha. Desde los primeros años de la década de 2000, esto significó la formulación de reformas que buscaban no sólo generar impactos en la administración, pero también en la doctrina y en la cultura institucional de las gestiones. Sin embargo, en la mayoría de los casos, los experimentos fueron incipientes y sus resultados, variados o incluso contradictorios. Por ejemplo, varios países de América Central siguen incumbidos en una verdadera guerra contra la delincuencia, y en países como Argentina, México y Perú se puso a los militares para combatir el narcotráfico sin una profunda planificación (Lagos y Dammert, 2005:55).

Se puede afirmar que, en la implementación de estos planes, se desconsideraron situaciones concretas como la negociación, la disensión, las contradicciones políticas y burocráticas. La nueva administración era "participativa en el nivel de discurso, pero centralizada en lo que respecta al proceso de toma de decisiones», y se "enfatizaron más las dimensiones técnicas que las dimensiones sociales y políticas de la gestión» (Paes de Paula, 2005:46). Además, la ambigüedad y la falta de claridad de los objetivos de muchos Planes, bien como los problemas de coordinación intergubernamental y los recursos limitados, se sumaron al hecho que «se daba poco valor a la recolección y al procesamiento de la información sobre el crimen» (Frühling, 2008:2). Esto incluso ocasionó limitaciones cogniti- 
vas, que por sí solas obstaculizaron los esfuerzos de modernización de la administración pública. En la práctica, fue imposible trabajar con todas las variables de la criminalidad y comprender su contingencia. «La fidelidad en la ejecución de diseños propuestos es siempre incompleta, en vista de la racionalidad, y porque la sincronía es siempre inexacta» (Velarde et al, 2007:48). Justamente por esto sería necesario un "ciclo vivo" y retro-alimentar de políticas públicas, de modo a corregir y superar esas y otras limitaciones en la implementación de los planes de seguridad.

Todavía, cuando se superaban las limitaciones cognitivas, la presión para conseguir resultados frente a la criminalidad podría traer efectos colaterales. Por ejemplo, en la «reforma modelo» del sistema penal de Chile iniciada en el 2002, la capacidad de respuesta del sistema penal llegó a ser de 89\% para resolver procesos judiciales (Duce, 2008: 74). Pero si por un lado hubo avances en la productividad y en el tratamiento de las víctimas, por otro lado, la reforma penal fue acompañada por una creciente sobrepoblación de las cárceles, por el crecimiento de los índices de reincidencia criminal y por la escaza planificación en la reinserción social de los condenados (Dammert, 2005:44).

Otro ejemplo es Minas Gerais. En 2003, el gobierno de este estado brasileño unificó los departamentos de Seguridad y Justicia, insiriéndolos en la «Secretaría de Estado y de Defensa Social» (SEDS) con el fin de mejorar la coordinación de sus instituciones en materia de seguridad pública. En el año 2008, la Secretaría implementó un sistema de metas en el que cada comisario de policía (delegado) fue obligado a realizar informes de al menos diez investigaciones criminales a cada mes. Como era esperado, hubo un aumento en el número de investigaciones de la policía, pero a menudo el informe de éstas era conducido de manera rápida, sin la debida investigación y resolución de los crímenes. Sumado a esto, las metas del sistema tampoco eran claras cuanto al modo de elaboración de los informes (Vargas y Nascimento, 2009:13-31).

Esos son sólo algunos ejemplos que se pueden extraer de las numerosas experiencias en las políticas de seguridad pública en América Latina. En estas, aunque se intentaron incorporar nuevos parámetros — como búsqueda de eficiencia, flexibilidad y gestión por metas- las medidas podían fallar no sólo en la internalización de esos parámetros, sino también en el deseo de dar continuidad a este tipo de acciones dentro de una política de estado que no fuese susceptible a las vicisitudes de un gobierno después de otro.

Por esas razones, se ha buscado una eficiencia y flexibilidad administrativas con el apoyo acciones que se pautan por la descentralización de tareas y por su carácter local. Es decir, reforzadas por el marco bottom-up de implementadores y destinatarios en las políticas, las "presiones de abajo» en la sociedad civil, junto a la participación de asociaciones privadas, tuvieron algunos efectos y dieron lugar a acciones como la policía comunitaria y la vigilancia entre vecinos (Dammert et al, 2004). Desde los años 90, sobre todo cuando iniciativas de las administraciones centrales eran incipientes, se desplegaron estrategias como el 
Plan Cuadrante de Carabineros en Chile, o las experiencias de policía comunitaria en Villa Nueva, Guatemala, en Bogotá, Colombia, y en Belo Horizonte, Brasil (Frühling, 2003). En los últimos años, también se han implementado acciones contra la violencia de género como la iniciativa «ciudad mujer» en El Salvador, o como la reformulación de las policías para fortalecer sus vínculos con las ciudanía en Quito, Ecuador (Martín Cubel, 2016). Tales experiencias fueron llamativas por su innovación y por mezclar lo «glocal» y la necesidad de aumentar la confianza en la labor policial.

No obstante, las prácticas de policía comunitaria han sido dependientes de las vicisitudes de los gobiernos locales, sobre todo en aquellas que se iniciaron en la década de 1990 a ejemplo de Belo Horizonte. Por otra parte, el suceso de estas prácticas ha sido limitado por su alcance geográfico y ha dependido de cambios nacionales. Por ejemplo, el hecho de que los homicidios en ciudades como Cali y Medellín se hayan reducido considerablemente, sobre todo si comparados a los índices de inicio del siglo, también debe ser entendido dentro del contexto de combate a grupos armados por parte del gobierno central y al subsecuente proceso de Paz. Además, las prácticas de policía comunitaria han dependido de la participación ciudadana, algo que pierde calidad y se desestimula en regiones donde la criminalidad se hace muy presente (Frühling, 2009).

Por lo tanto, pese a las acciones prometedoras de la policía comunitaria, el cambio de una política de seguridad con énfasis en la represión para otra con visión más integral o del "ciclo vivo" -que por ejemplo incluyera acciones sociales de prevención del delito-, bien como el cambio hacia una seguridad más ciudadana, pueden ser resumidos a un conjunto heterogéneo de acciones que aún tienen que ajustar sus bases y desarrollo, sobre todo si se considera que los índices de homicidios y delincuencia aun representan un obstáculo para el perfeccionamiento de las democracias en los último años.

En el caso de los homicidios, las tasas persisten como críticas en Honduras, Belice, El Salvador, Guatemala, Colombia y, recientemente, Venezuela (grupo donde hay más de 30 muertes por cada 100.000 habitantes). El índice también es elevado en México, Brasil y República Dominicana (con tasas entre 20 a 30), así como en Ecuador, Bolivia, Nicaragua, Panamá, Guyana y Guyana Francesa (con tasas entre 10 a 20). Por otra parte, este tipo de crimen tiene menor incidencia en Perú, Paraguay, Costa Rica, Argentina, Uruguay (con tasas de 5 a 10) y sobre todo en Chile y Cuba (tasas entre 3 a 5) según datos del último estudio de UNODC en 2013. En términos temporales, la tasa de homicidios se ha incrementado durante la última década sobre todo en los primeros grupos. A su vez, el panorama contempla otros países como Nicaragua, Argentina, y Chile, donde se dan muestras de reducción o estabilidad de dichas tasas (Martín Cubel, 2016). No obstante, en los grupos con menores tasas de homicidios aún se enfrentan elevadas tasas de otros crímenes, como robos seguidos de violencia, sobre todo si se comparan con otras regiones del mundo (PNUD, 2014). Finalmente, existe una heterogeneidad en términos sub-nacionales y un desplazamiento de la criminalidad hacia zonas menos urbanizadas como en el caso de Brasil, donde apenas $26 \%$ de los más de 50 mil homicidios del año 2016 se cometieron en las ca- 
pitales o grandes ciudades. ${ }^{9}$ En suma, hasta mediados de la segunda década del siglo, «la región ha sido escenario de dos grandes expansiones, la económica pero también la delictiva» (PNUD, 2014).

Por todo ello, se infiere que, desde el final del conflicto bipolar en el siglo pasado, se ha avanzado en reformas que buscan adaptar instituciones, normas y procesos de acción administrativa para tratar el fenómeno de la criminalidad. Pero también hubo falta de visión sistémica (como en la reforma de las instituciones relacionadas con la acción policial), limitación de los cambios gerenciales (como en la definición e implementación de planes nacionales) y dificultades en los intentos de inclusión de nuevos actores extra-estatales (como en la policía comunitaria). A groso modo, las políticas han tenido carácter más superficial, siendo limitadas si se piensa que estas podrían promover nuevas perspectivas y mejorar los resultados en el tratamiento de la seguridad pública en América Latina. Por lo tanto, se prolonga un panorama de desajuste entre las políticas de seguridad y las respuestas hacia al fenómeno de la criminalidad, en mayor o menor grado, dentro de cada país de la región.

\section{CONCLUSIÓN}

Una región, cuyas políticas públicas de seguridad pública se han presentado desajustadas tanto en relación a sus bases como en su modo de acción frente a la criminalidad, puede terminar promoviendo tal fenómeno. Básicamente, eso es lo que se verificó del panorama común de la trayectoria de muchos países latinoamericanos durante las últimas décadas. El recurso a la violencia y al crimen entre los ciudadanos, así como la incapacidad política y social de los Estados para mediar y resolver conflictos, sugieren que se ha producido una contingencia en la cual se han formulado respuestas insatisfactorias.

En el pasado reciente de América Latina, en escenarios posteriores al fin de dictaduras, guerras civiles o conflictos internos, se tuvo el anhelo de llegar a mejores condiciones de paz y ciudadanía, pero este ha sucumbido, entre otros factores, ante las inconsistencias de las propias políticas públicas. Sin herramientas que permitiesen profundizar el entendimiento de las políticas frente a la criminalidad, fue necesario fortalecer las capacidades institucionales para erigir y remodelar las políticas de seguridad pública. Sin embargo, estas se limitaron a enfoques policiales y punitivos en sociedades marcadas por la pobreza, la desigualdad social y con considerables índices de impunidad.

Al mismo tiempo, las salidas locales como la policía comunitaria, aunque hayan traído un enfoque prometedor frente al crimen, han perdido ímpeto desde sus inicios o presentan limitaciones de alcance político y geográfico. Por tales factores, se han verificado desajustes entre una acción pública y sus consecuencias, sobre todo si se considera que las tasas

\footnotetext{
${ }^{9}$ Anuario Brasileiro de Segurança Pública, 2016. Disponible en: http://www.forumseguranca.org.br/storage/ download//anuario-2016-03nov-final.pdf, consulta en 15 de Noviembre de 2016.
} 
de criminalidad, y dentro de ellas los homicidios, han aumentado desde el inicio del siglo en lugares como Honduras, Belice, El Salvador, Guatemala y México. El mismo sigue representando un desafío de primer orden en Colombia, Ecuador, Bolivia, Caribe y ha presentado un desplazamiento sub-nacional y fuerte incidencia en países como Brasil. Mientras tanto, otros crímenes como los robos seguidos de violencia siguen siendo relevantes en Perú, Argentina, Chile y Uruguay.

En las últimas décadas, numerosas redes de criminalidad han aprovechado tanto la relativa debilidad de los estados para formular y ejecutar políticas, como de la relativa complicidad de estos con aquellos a través de la corrupción. Estas redes, a su vez, han desafiado las agendas nacionales e internacionales de la región. En este sentido y frente a una idea generalizada, el Estado débil proporciona más ventajas al crimen organizado que un Estado fallido (Alda Mejías, 2015). Concomitantemente, para algunos ciudadanos que viven en condiciones en las que la protección estatal se encuentra deficitaria, el uso de la violencia no necesariamente se ha considerado como un medio para un fin ilegítimo (Landman, 2010). Todo ello en una era donde la seguridad, privilegio para pocos, también puede ser comprada por aquellos capaces de pagarla. De modo que las políticas van cediendo paso a una «gobernanza» que se efectúa por redes del crimen y otros actores no-estatales, y con todos los problemas que esto puede representar.

La gobernanza efectuada por «redes de poder paralelas» amplifica lo que Guillermo O’Donnell (1977) llamaba «autoritarismo burocrático». Es decir, se amplían los vínculos que sostienen intereses materiales y simbólicos entre burócratas estatales e agentes privados (sean del mundo del crimen o de entidades corporativas), los cuales se presentan ajenos a cambios populares y democráticos. Tales redes son un elemento central en la cuestión de la seguridad, pues estas permean, conviven y ejercen presión adicional a las políticas públicas. En última instancia, este tipo de redes puede obstruir cualquier camino de democratización y de acción contra el crimen.

Pero lo positivo es que la lógica de las redes también puede calibrar el control y el tratamiento de la criminalidad con mejores acciones de corte integral. Por ejemplo, sin la coordinación entre la policía, las prisiones y el sistema judicial, o sin la participación de la sociedad organizada es difícil pensar en términos de eficiencia dentro de las acciones dirigidas al crimen y la violencia en los espacios de convivencia colectiva. Como aprendido en los modelos bottom up y del "ciclo vivo», se debe tener en cuenta que las bases de las políticas públicas huyen a una planificación a priori y a la pura centralización de decisiones. Por lo tanto, una lógica de redes también puede integrar el aprendizaje de modelos de políticas públicas preocupadas con implementadores y destinatarios.

Parte del aprendizaje también es reconocer que, en las presiones de actores políticos estatales y extra-estatales, en las demandas colectivas y en los mecanismos de implementación de decisiones, se produce un campo de complejidad para la acción pública. En este campo, las acciones no se ajustan a la nitidez de los planes, a las normativas institucionales y a la 
intervención en la realidad social. Se necesitaría, por lo tanto, inserir las políticas originalmente formuladas, las acciones valorativas con otros actores, los mecanismos de implementación y evaluación, dentro de un «ciclo vivo» retroalimentar. Un ciclo donde se reajustan las políticas junto a otros sectores (issues), se corrigen los errores y se potencializan los resultados obtenidos a favor de la ciudadanía.

Mientras tanto, se ha expuesto que, en el devenir reciente y actual de las políticas de seguridad en América Latina, se han producido desajustes en las bases y en las acciones gubernamentales frente a la criminalidad. Tales desajustes, a su vez, constituyen un obstáculo adicional para profundizar la democracia y para atender necesidades materiales y humanas. Al mismo tiempo, el crimen, en sus más variadas formas y motivaciones, aún sigue siendo un elemento de primer orden en la historia actual de la región.

\section{REFERENCIAS BIBLIOGRÁFICAS}

Alda Mejías, S. (2015). La debilidad del imperio de la ley en América Latina: Un factor para entender la implementación del crimen organizado. Revista Española de Ciencia Política, num. 37. Marzo 2015, pp- 63-88.

Alda Mejías, S. y Sousa Ferreira, S. (2015). La multidimensionalidad de la seguridad nacional: retos y desafíos de la región para su implementación. Instituto Universitario General Gutierrez Mellado de la Investigación sobre la paz, la seguridad y defensa. Madrid: Imprensa AEBOE.

Beato Filho, C. C. (1999). Políticas Públicas de Segurança e a Questão Policial: Eficiência, equidade e accountability. In: Marcus André Melo. (Org.). Reforma do Estado e Mudança Institucional no Brasil. Recife: Fund. Joaquim Nabuco e Editora Massangano, v. 1, p. 335-65.

Beliz, G.(2012). Gobernar la seguridad ciudadana en América Latina y el Caribe: Amenazas, desafíos y nudos estratégicos de gestión. Banco Interamericano de Desarrollo. Sector de Instituciones para el Desarrollo. Disponible en: https://publications.iadb. org/bitstream/handle/11319/5100/Gobernar\%20la\%20seguridad\%20ciudadana\%20 en $\% 20 \mathrm{Am} \%$ C3\%A9rica\%20Latina $\% 20 y \% 20$ el\%20Caribe.pdf?sequence=1, consulta en $09 / 08 / 2016$.

Borges, N.. (2003). A Doutrina de Segurança Nacional e os governos militares. In: O Brasil Republicano. Jorge Ferreira e Lucilia Almeida (org.), Rio de Janeiro: Civ. Brasileira.

Buvinic, M. y Morrison, A. (1999). La violencia en América Latina y el Caribe: un marco de referencia para la acción. Washington D.C.: División de Desarrollo Social, Banco Interamericano de Desarrollo. 
Caruso, H. et AL. (2007). Polícia, estado e Sociedade: Práticas e Saberes Latino-americanos. Rio de Janeiro: Ed. Publit Soluções.

Comisión Económica para América Latina y el Caribe, CEPAL (2011). Panorama Social de América Latina. Comisión Económica para América Latina y el Caribe. Disponible en <http://www.cepal.org/publicaciones/xml/3/45173/2011-820- PSP-Sintese-Lanzamiento.pdf $>$ consulta en 14/07/2015.

- (2015). Panorama Social de América Latina 2015. Disponible en <http://www.cepal. org/es/publicaciones/panorama-social-america-latina-2015-documento-informativo> consulta en 9/11/2016.

Cerqueira, D. y Carvalho, J. L. (2007). O jogo dos sete mitos e a miséria da segurança pública no Brasil. In: Marcus Vinicius Gonçalves da Cruz; Eduardo Cerqueira Batitucci. (Org.). Homicídios no Brasil. 1. aed. Rio de Janeiro: Editora FGV, v. 1, p. 1-252.

Dammert, L. et. al. (2004). Seguridad ciudadana: experiencias y desafíos. Valparaíso: I. Municipalidad de Valparaíso, Red 14, Programa URB-AL, Azevedo.

Dammert, L. (2005). Violencia Criminal y Seguridad Ciudadana en Chile. Santiago de Chile: División de Desarrollo Social, CEPAL.

Dammert, L. (2007). Dilemas da Reforma Policial na América Latina. In: Polícia, estado e Sociedade: Práticas e Saberes Latino-americanos, Caruso, H.; Muniz, J.; Blanco, A. C. C. (org.), Rio de Janeiro: Publit Soluções Editoriais.

Dammert, L. y Bailey, J. (2005). Seguridad y Reforma Policial en las Américas: Experiencias y desafíos. Lucia Dammert \& John Bailey (org.), México D.F.: Ed. Siglo Veintiún. Dosse, François (2012). História do tempo presente e Historiografia. In: Revista Tempo e Argumento. Vol. 4. Florianópolis, 2012, p.14.

Dammert, L. (2014). Dos décadas de reformas policiales en América Latina. Lecciones y desafios. In: Violencia, delincuencia y seguridad pública en América Latina. Org: Maihold, G. y Córdova M., R. Ciudad de México: Editorial Cenzontle, SA.

Duce, M. (2008). La Reforma Procesal Penal en Chile: Logros y Desafíos, Período 2000-2007. Urvio, Revista Latinoamericana de Seguridad Ciudadana. N. 3. FLACSO, Quito, pp. 67-84.

Espinoza, H. (2008). Hacia una Visión Político Progresista en Seguridad Ciudadana. Seminario Internacional. Santiago de Chile: ProSur.

Frühling, H. (2003). Policía Comunitaria y Reforma Policial en América Latina ¿Cuál es el impacto? Centro de Estudios en Seguridad Ciudadana. Universidad de Chile, Serie de Documentos, Santiago de Chile. 
Frühling, H. (2008). Sistema de Seguridad Pública en América Latina: Casos comparados. En Comunidad más Prevención. Boletín N. ${ }^{\circ}$ 7, Santiago: CESC.

Frühling, H. (2009). Violencia y policía en América Latina. Facultad Latinoamericana de Ciencias Sociales (FLACSO Sede Ecuador), Quito.

Garland, D. (1996). The Limits of the Sovereign State: Strategies of Crime Control in Contemporary Society. The British Journal of Criminology, vol. 36, n. 4.

Garretón, M. A. (1997). Revisando las transiciones democráticas en América Latina. Revista Nueva Sociedad, N. 148.

Herbert, S. (1999). The End of Territorially-sovereign State? The Case of Crime Control in the United States. Political Geography, vol. 18, n.2.

Hernández Milian, J. (2008). Mapeo del caso Centroamericano. In: El crimen organizado en América Latina y el Caribe: Amenazas y Perspectivas. Ciudad de México: Fund. Friedrich Ebert.

Kingdon, J. (1984). Agendas, Alternatives, and Public Policies. Boston: Little, Brown. Lagos, Roberto (2012). Política de seguridad, La Experiencia Chilena: (2003-2011). Revista Análisis público. Universidad de Valparaíso. Año 1, N. 1, 2012, pp. 211-232.

Lagos, M.; y Dammert, L. (2012). La Seguridad Ciudadana: El problema principal de América Latina. Latinobarómetro. Lima.

Lahera, E. (2002). Introducción a las Políticas Públicas. Santiago: FCE.

Landman, T.. (2010). Violence, Democracy and Human Rights in Latin America. In: Violent Democracies in Latin America, ed. by Enrique Desmond Arias, Daniel Goldstein, 226-241. Durham and London: Duke University Press.

Latinobarómetro (1995-2015). Informes y análisis. Disponibles en < http://www. latinobarometro.org/latOnline.jsp> acceso en Nov. 2015.

Lindblom, Ch. (1991). El Proceso de Elaboración de las Políticas Públicas. España: Ministerio para las Administraciones Públicas.

Linz, J. y Stepan, A. (1996). Problems of Democratic Transition and Consolidation: Southern Europe, South America and Post-communist Europe. Baltimore: The Johns Hopkins University Press.

Maihold, G.y Córdova Macías, R. (2014). Violencia, delincuencia y seguridad pública en América Latina. Ciudad de México: Editorial Cenzontle, S.A. 
Martín Cubel, Fernando (2016). El estado de la seguridad en América Latina. Instituto Español de Estudios Estratégicos. Documento Opinión, Enero de 2016.

Martínez, J. (1990). Violencia social y política en Santiago de Chile. (1947-1987). In: Personas y escenarios en la violencia colectiva. Santiago: Ediciones SUR.

Meny, I.; Thoenig, J. (1992). Las políticas públicas. Barcelona: Ariel.

Muller, P. (2002). Las Políticas Públicas, Bogotá: Universidad Externado de Colombia.

O'Donnell, G. (1977). Corporatism and the Question of the State. In: Authoritarianism and Corporatism in Latin America, edited by James Malloy, 47-87. University of Pittsburgh Press.

O’Donnell, G.(1994). Delegative Democracy. Massachusetts, Journal of Democracy, pp. 55-69.

Orrego, C. (1998). Modernización del Estado y la gestión pública en Chile: balance y desafíos. Seminario Presente y futuro de los procesos de reforma del Estado. Montevideo.

Oviedo, E. (2002). Democracia y seguridad ciudadana en Chile. In Violencia, Sociedad y Justicia en América Latina. Buenos Aires: Clacso. Disponible en <http://biblioteca. clacso.edu.ar/ar/libros/violencia/oviedo.pdf> acceso en Ago. 2014.

Paes de Paula, A. P. (2005). Administração Pública Brasileira entre o Gerencialismo e a Gestão Social. RAE (Impresso), São Paulo, v. 45, n.1, p. 36-49. Disponible en<http://www. scielo.br/pdf/rae/v45n1/v45n1a05.pdf> acceso en Oct. 2014.

Padros, E. (2012). A ditadura civil-militar uruguaia: doutrina e segurança nacional.

Varia hist., Belo Horizonte, v. 28, n.48, disponible en <http://www.scielo.br/scielo. php?script=sci_arttext\&pid=S0104- 87752012000200002\&lng=en\&nrm=iso $>$. Consulta en $19 / 06 / 2015$.

Programa de las Naciones Unidas para el Desarrollo, PNUD (1998). Desarrollo humano en América Latina, Las paradojas de la modernización, Santiago de Chile: PNUD.

- (2010). Informe Regional sobre Desarrollo Humano para América Latina y el Caribe. N. 2, p. 26-62.

- (2014). Informe Regional sobre Desarrollo Humano. Seguridad Ciudadana con rostro humano: Diagnóstico y Propuestas para América Latina. Disponible en: http://www. undp.org/content/dam/rblac/img/IDH/IDH-AL\%20Informe\%20completo.pdf, consulta en 11/08/2016. 
- (2016). Informe Regional sobre Desarrollo Humano. Progreso multidimensional: Bienestar más allá del ingreso. Disponible en: http://www.latinamerica.undp. org/content/rblac/es/home/library/human_development/informe-regional-sobredesarrollo-humano-para-america-latina-y-e.html, consulta en 23/09/2016.

Quiroga, H.(2005). El tiempo del 'proceso'. In: Dictadura y democracia (1976-2001). Juan Suriano (Director), Buenos Aires: Sudamericana.

Ramírez, H. (2012). Política e História do tempo presente na historiografia das ditaduras do Cone Sul na América Latina. Revista Tempo e Argumento. Florianópois, Vol. 4.

Sanseviero, R. (2007). Entre las intenciones y las tensiones. In: S. Escobar y J. Ensignia. (ed.), Seguridad Pública en los países del cono sur. Los desafíos institucionales, Santiago de Chile, Fundación Friedrich Ebert.

Silva, P. y Melo, M. (2000). O processo de implementação de políticas públicas no Brasil: características e determinantes da avaliação de programas e projetos. Campinas: NEPP/ UNICAMP, Cuaderno 48.

Souza, Luís (2011). Políticas Públicas e a área da segurança no Brasil. Debate em torno de um novo paradigma. Caxambu-MG: $35 .^{\circ}$ Encontro Nacional da Anpocs.

Sozzo, M.(2005). Metamorfosis de los Discursos y las Prácticas sobre Seguridad Urbana en la Argentina. In: Seguridad y Reforma Policial en las Américas: Experiencias y desafíos. Lucia Dammert \& John Bailey (org.), México D.F.: Ed. Siglo Veintiún.

Subirats, J.; Knoepfel, P.; Larrue, C. y Varone, F. (2008). Análisis y gestión de políticas públicas, Madrid: Ariel.

Tudela, P.(1998). Prevención del delito y seguridad ciudadana en democracia. In: Cuaderno de Criminología. Santiago: Instituto de Criminología de la Policía de Investigaciones de Chile, N. $^{\circ} 8$.

UNODC (2011). World Report on Homicides. United Nations Office on Drugs and Crime. Disponible en <http://www.unodc.org/documents/data-and- analysis/statistics/ Homicide/Globa_study_on_homicide_2011_web.pdf> consulta en Nov. 2014.

- (2013). World Report on Homicides. United Nations Office on Drugs and Crime. Disponible en < http://www.unodc.org/documents/gsh/pdfs/2014_GLOBAL_HOMICIDE_ BOOK_web.pdf > consulta en Nov. 2016.

Valenti, G. y Flores, U. (2009). Ciencias sociales y políticas públicas, In: Revista Mexicana de Sociología, núm. 71 (especial), México: UNAM, pp. 167-191. 
Criminalidad y desajustes de las políticas de seguridad... | Jaseff Raziel Yauri Miranda

Vargas, J. y Nascimento, L. (2009). O inquérito Policial no Brasil - Uma Pesquisa Empírica: O caso da investigação criminal de homicídios dolosos em Belo Horizonte. In: Cadernos Temáticos da CONSEG. Brasília: Coordenação Geral da 1. ${ }^{a}$ Conferência Nacional de Segurança Pública Ministério da Justiça - Ano I, n. 06.

Velarde, J. et al. (1997). Entre el diseño y la evaluación: el papel crucial de la implementación de los programas sociales. Banco Interamericano de Desarrollo, Washington: BID Press. 